\section{Cahiers balkaniques}

36-37 | 2008

L'image de la période ottomane dans les littératures balkaniques

\title{
Les Turcs dans Ţiganiada de Ion Budai-Deleanu
}

Turcs in Tुiganiada of Ion Budai-Deleanu

\section{Silviu Petcu}

\section{(2) OpenEdition}

1 Journals

Édition électronique

URL : https://journals.openedition.org/ceb/1543

DOI : $10.4000 /$ ceb. 1543

ISSN : 2261-4184

Éditeur

INALCO

\section{Édition imprimée}

Date de publication : 1 décembre 2008

Pagination : 231-241

ISBN : 978-2-85831-173-6

ISSN : 0290-7402

\section{Référence électronique}

Silviu Petcu, «Les Turcs dans Țiganiada de Ion Budai-Deleanu », Cahiers balkaniques [En ligne], 36-37 | 2008, mis en ligne le 16 mai 2012, consulté le 06 juillet 2021. URL : http://journals.openedition.org/ ceb/1543; DOI : https://doi.org/10.4000/ceb.1543

Ce document a été généré automatiquement le 6 juillet 2021.

\section{(c) (†) \&}

Cahiers balkaniques est mis à disposition selon les termes de la Licence Creative Commons Attribution - Pas d'Utilisation Commerciale 4.0 International. 


\section{Les Turcs dans Ţiganiada de Ion Budai-Deleanu}

Turcs in Tुiganiada of Ion Budai-Deleanu

\section{Silviu Petcu}

1 La Tiganiada est une épopée placée dans la Munténie ${ }^{1} \mathrm{du} \mathrm{XV}^{\mathrm{e}}$ siècle et structurée sur deux plans : d'une part un plan comique créé par le pittoresque du campement des Rroms, appelés "Tsiganes ", mobilisés pour combattre les Turcs par le prince Vlad l'Empaleur ${ }^{2}$ (Vlad Ţepeş, plus connu sous le surnom de Dracula ${ }^{3}$ - surnom dont il avait hérité de son père ${ }^{4}$, ainsi nommé par les Allemands) et d'autre part un plan héroïque constitué par les actions de ce prince empaleur ${ }^{5}$ et de son armée. La promesse d'émancipation des Rroms esclaves, avec attribution d'un territoire, en échange de leur alliance au combat est une pure fiction et même un anachronisme pour le $\mathrm{XV}^{\mathrm{e}}$ siècle. Mais à travers cette idée, Budai-Deleanu ${ }^{6}$ se révèle visionnaire et aussi partisan du mouvement d'abolition de l'esclavage des Rroms qui n'arrivera à ses fins que le 20 février 1856 (8 février du calendrier orthodoxe). L'anecdote de l'enrôlement des Rroms par Vlad pour étoffer ses troupes se retrouve sous diverses formes dans les pamphlets en latin et allemand de Matthias Corvin ${ }^{7}$ et a été étudiée par le professeur Matei Cazacu, qui la qualifie de « farce sinistre ${ }^{8} »$.

2 Tout au long de son œuvre, Budai-Deleanu insère nombre d'éléments allégoriques qui lui donnent la possibilité de passer avec facilité de l'événement historique réel à un monde imaginaire de saints et de démons. Dans son acception, ceux-ci sont les uns partisans des chrétiens et les autres partisans des musulmans. Épopée héroïco-comique et satirique, la Ţiganiada doit être appréciée avant tout comme une allégorie où le plan de la fiction remplace souvent le plan réel. Mais l'auteur nous présente aussi le plan réel avec ses particularités historiques et nationales. Ion Budai-Deleanu, comme beaucoup d'autres auteurs chargés d'un idéal patriotique confirme la lutte sans cesse recommencée des pays roumains contre la puissance ottomane. N'oublions pas qu'il a été aussi un des pères de la langue roumaine moderne. 
3 L'auteur s'arrête sur une page représentative de l'histoire roumaine, celle de la période du prince Vlad III de Munténie. Ce souverain peint par les chroniques de l'époque notamment les pamphlets de Mathias Corvin, comme un monstre sanguinaire, est surnommé Vlad l'Empaleur en raison de son faible pour cette forme de châtiment: l'empalement. Selon les sources, il a régné pendant trois périodes ${ }^{9}$ : l'automne 1448 , puis 1456-1462 et enfin novembre-décembre 1476. Sa vie a été pleine de tourments : il a été otage chez les Turcs entre 1442 et 1448 en échange de la liberté de son père Vlad le Diable, qui avait été fait prisonnier par le sultan; entre 1449 et 1456 il a été en exil en Moldavie et Transylvanie, puis entre 1462 et 1474 a été le prisonnier du roi Matias Corvin dans la prison de Višegrad «située à 4 jours de Buda ${ }^{10}$ ». Conformément aux rapports de Thomasiis, Vlad III de Munténie a été arrêté par le condottiere tchèque Jan Jiskra de Brandys ${ }^{11}$. En 1474, après 12 ans de détention, Vlad III est libéré par le roi Matthias Corvin pour combattre contre les Turcs qui ont attaqué la ville d'Oradea, le 7 février $1474^{12}$. Vlad III de Munténie meurt assassiné par les boyards asservis aux Turcs, à la fin du mois de décembre 1476, à Bucarest (ou au début de janvier 1477) ${ }^{13}$.

4 En raison de son insoumission entêtée au sultan Mahomet II ${ }^{14}$, Vlad l'Empaleur devait rester dans l'histoire comme un patriote qui voulait protéger son pays contre l'expansion ottomane - même s'il est plus connu à l'étranger sous la caricature d'un vampire. En réalité, le courage et les capacités stratégiques de Vlad sont remarquables, surtout si l'on tient compte aussi de la valeur et de la force de son adversaire - mais aussi de sa cruauté. En plus de la grande force militaire et financière dont disposait l'Empire ottoman, existait aussi un effet psychologique induit par la grande renommée du sultan Mahomet II. C'était en effet lui qui avait réussi à donner le coup de grâce à l'Empire byzantin en 1453. Il occupait donc Constantinople et le monde chrétien tremblait devant ce sultan puissant et redoutable. Par rapport à l'armée de Vlad, l'armée du sultan Mahomet II était formée en grande partie de soldats professionnels, ce qui donnait au sultan l'avantage d'avoir une armée plus entraînée et aguerrie.

5 Ion Budai-Deleanu, remarquable érudit, est notamment un fin connaisseur des chroniqueurs L. Chalcocondili et M. Doukas. Les Expositions historiques de Laonic

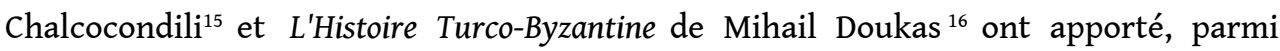
d'autres sources d'information, des données importantes pour l'œuvre de BudaiDeleanu en ce qui concerne l'expédition de Mahomet II contre Vlad l'Empaleur.

Des émissaires, envoyés en secret par les boyards infidèles, informent le sultan que Vlad prépare une rébellion contre l'Empire ottoman et lui demandent de se dépêcher pour détourner à temps les actions du prince régnant de la Munténie (chant III, strophe 98). En secret, le sultan Mahomet II rassemble ses commandants et leur ordonne de commencer tout de suite des pillages continus "sans cesse, hiver et été » en Munténie (chant III, strophe 99). Mais Vlad est informé que des troupes turques sont entrées en Munténie. Les troupes turques sont vaincues et les Turcs empalés vifs (presque 30.000 soldats) par Vlad et son armée (chant III, strophes $100-101)^{17}$.

7 Le sultan est si surpris de cette information qu'il ne veut pas y croire. Il la fait vérifier par des personnes de confiance de son entourage. Mahomet II veut arrêter Vlad et pour faciliter ce plan met en scène une invitation du prince roumain à Istanbul pour apporter personnellement le tribut ${ }^{18}$. Le sultan envoie son secrétaire Catavolinos ${ }^{19}$ avec la mission d'inviter le prince Vlad à Istanbul. En même temps, le sultan ordonne en secret à Hamza le pacha de Vidin, d'essayer d'arrêter par tous les moyens le prince Vlad quand il accompagnera Catavolinos à Istanbul (chant III, strophes 102 - 109). 
8 Vlad reçoit le messager qui lui fait savoir - sans doute par un firman -, que toutes les erreurs du passé seront pardonnées par l'immense magnanimité du sultan et lui propose la totale amitié de la Sublime Porte. Le sultan lui demande seulement de se présenter en tant qu'ami à sa cour pour lui apporter le tribut et 500 jeunes garçons ${ }^{20}$ (chant III, strophes 110 - 116).

9 Le messager du sultan comprend qu'il ne peut pas convaincre le prince de la Munténie d'aller à Istanbul et annonce à Hamza de se préparer pour donner suite au plan du sultan. Catavolinos demande à Vlad de l'accompagner jusqu'au bord du Danube (chant III, strophes 117 - 118).

10 Vlad est prévenu par ses espions du danger de tomber dans un piège. Il envoie en secret 4.000 cavaliers pour attendre Hamza et ses troupes ${ }^{21}$ et accompagne Catavolinos, sans mot dire et sans avoir avec lui plus de soldats que sa garde habituelle (118-120). Arrivé dans l'endroit choisi par les Turcs, le cortège du prince Vlad est attaqué comme prévu par Hamza et ses 10.000 soldats. Les Turcs pensent avoir réussi à arrêter Vlad quand celui-ci donne aux troupes qui attendaient cachées l'ordre de passer à l'attaque et luimême se lance courageusement avec sa garde dans la mêlée où il frappe les Turcs « comme une foudre ». Le combat finit par la victoire de Vlad. Il fait empaler Hamza le pacha de Vidin avec ses troupes et Catavolinos, ${ }^{22}$ le secrétaire du sultan, sur des pals plus hauts que la moyenne (chant IV, strophes 25 - 41).

11 Personne dans l'entourage du sultan n'a le courage de lui annoncer la triste nouvelle. Son vizir prend sur lui de le faire et lui apprend le déroulement des événements. Le sultan en colère punit le malheureux vizir et ordonne qu'une grande armée soit prête au printemps (chant IV, strophes 43 - 47). Budai-Deleanu, en tenant compte de ses sources, évalue cette armée à 200.000 soldats envoyés par voies terrestres et maritimes ${ }^{23}$.

12 L'armée du sultan arrive à Vidin au milieu du printemps et la nouvelle de l'invasion effraie la population roumaine qui se cache dans les montagnes. Vlad prépare son armée pour une lutte de harcèlement et, chaque fois que l'occasion se présente, il attaque les troupes turques. Il profite de l'avantage du terrain pour se cacher et suivre l'armée ottomane "tel un loup affamé qui chaque fois qu'il voit une brebis égarée, l'attrape et s'enfuit avec elle ».

13 Le sultan occupe villes et villages et arrive jusqu'aux montagnes. Il comprend la stratégie de Vlad et décide de changer de tactique. Durant cette guerre de guérilla, dans un pays désolé, les troupes turques commencent à se démoraliser et donnent des signes alarmants de fatigue. Le sultan ordonne alors le campement de l'armée et envoie des émissaires à Bucarest pour annoncer aux boyards qu'il veut seulement punir le prince Vlad, mais qu'il entend bien pardonner au reste du pays. Il espère ainsi diviser les Roumains et affaiblir leur loyauté envers Vlad (chant IV, strophes 48 - 57, chant VII, strophes 16 - 19).

14 Vlad comprend l'idée du sultan et à son tour décide de mettre en application un plan courageux. Il change ses vêtements et s'introduit dans le campement de l'armée turque pour s'informer sur la manière d'attaquer avec le plus de chances de réussite (chant VII, strophes 20 - 25).

15 Le courage certes, mais aussi la terrifiante perspective de l'empalement, déterminent les soldats du prince Vlad à ne pas le trahir même si l'ennemi menaçait de les tuer ${ }^{24}$ (chant VII, strophes 54 - 59). 
16 Vlad choisit la nuit du 17 juin 1462 pour l'attaque. Cette nuit est restée célèbre pour son originalité dans l'histoire militaire roumaine. En effet, avant le prince Vlad l'Empaleur, aucun autre prince roumain n'avait attaqué l'ennemi de nuit.

Budai-Deleanu écrit que Vlad a attendu la tombée de la nuit pour sortir avec son armée organisée en trois colonnes. Il avait décidé d'attaquer depuis trois directions pour empêcher les troupes turques de se rassembler. L'attaque commence en silence avec pour objectif de faire le plus de dégâts possible dans l'armée turque. Les Ottomans sont attaqués en plein sommeil. Dans le campement turc règne alors le chaos. Pour donner l'impression aux Turcs d'être encerclés par une armée roumaine innombrable, le prince Vlad a dissimulé dans les buissons qui entouraient le campement quelques centaines de soldats avec des cornes et des tambours et l'ordre de faire beaucoup de bruit. À la lumière des torches, l'armée roumaine avance dans le campement et met en fuite le corps d'armée asiatique du sultan. Pour obliger les soldats turcs à rester et à faire face à l'ennemi, les hérauts du sultan les menaçaient de mort s'ils quittaient leurs positions ${ }^{25}$ (chant VII, strophes 64 - 83).

18 La panique est si grande que malgré tout les Turcs quittent en désordre le campement, jusqu'au sultan qui s'enfuit entouré de ses gardes fidèles. Le campement plein de morts et de blessés tombe dans les mains des Roumains victorieux (chant VII, strophes 84 87).

19 Le sultan, avec ses gardes, se cache dans la forêt et envoie des cavaliers pour regrouper l'armée en fuite. C'est alors que fatigué, il s'endort et fait un cauchemar où il voit le spectre de Hamza pacha qui l'invite à le suivre. Le sultan voit des milliers de soldats turcs empalés. Il se réveille en sursaut et un serviteur doit le réconforter. Il tombe dans le désespoir et veut même se suicider, mais au dernier moment un de ses soldats lui apporte la nouvelle que des troupes regroupées sont sur le point d'arriver. Le sultan reprend courage et se hâte vers le Danube pour quitter la Munténie (chant VIII, strophes 107 - 119).

À l'exception des chroniqueurs turcs, toutes les autres sources parlent de la retraite précipitée et en désordre des troupes ottomanes, qui n'ont pas réussi à atteindre le but de leur expédition, celui de punir le prince de la Munténie.

21 L'archange Gabriel va alors rendre visite à Vlad pour lui annoncer que Dieu n'entend pas, sous son règne, libérer le pays du joug de la Sublime Porte: "les décisions éternelles restent inchangées, elles veulent que ton peuple reste encore longtemps sous la servitude païenne» (XII.99) ${ }^{26}$. Vlad prend alors le chemin de l'exil - selon d'autres sources il est arrêté par les Hongrois, laissant la destinée du pays entre les mains de Brâncoveanu. Celui-ci réunit ses hommes, lesquels, dans une scène magnifique, font renaître l'espoir aux derniers vers de l'épopée.

On sait que quelques années plus tard - à l'automne 1595, les Turcs sont repoussés au sud du Danube par le souverain de Moldavie, le célèbre Răzvan Vodă (ştefan Răzvan) qui malgré cette victoire est déchu puis empalé le 13 décembre de la même année, victime d'un complot turco-polonais.

23 Une fois de plus l'histoire des Roumains montre que par leur lutte opiniâtre, ils ont réussi à retarder l'avancée des Turcs vers l'Europe centrale. Ce retard a d'une part déterminé l'affaiblissement de l'offensive ottomane et d'autre part, laissé le temps nécessaire à l'Europe de l'ouest pour se préparer et livrer les batailles dans des périodes plus favorables et avec des armes plus puissantes et plus efficaces que celles des Turcs. 
La petite armée roumaine formée par des paysans et des boyards ne pouvait à elle seule arrêter la force ottomane. Elle a réussi seulement à la retarder avec héroïsme, mais ce retard fut décisif pour l'Histoire.

Le récit très factuel que nous présentons ici n'est qu'un des éléments de cette œuvre d'une exceptionnelle richesse qu'est la Ţiganiada. Pour une analyse plus détaillée, nous ne pouvons que renvoyer au long chapitre que lui consacre Marcel Courthiade dans Les Rroms dans les Belles-Lettres européennes ${ }^{27}$, où il met en évidence la complexité de cet impressionnant poème de près de 1400 sizains très réguliers répartis en douze chants. Au-delà de son sous-titre d'héroïco-comique, l'ouvrage rassemble en effet des réflexions qui gardent toute leur valeur de nos jours sur la politique, la religion, la fidélité, la gloire, la tendresse et même la dialectologie et la poétique. Comme on a pu le voir dans le survol donné plus haut, l'image des Turcs y est en tous points conforme à leur réputation largement répandue dans les Balkans où ils étaient attirés par la soif du haraç. Cruauté, perfidie, corruption et absence de compassion les caractérisent d'un bout à l'autre, mais l'ouvrage montre que ces maux n'auraient pu s'exercer sans les bassesses des boyards - voire le propre frère de Vlad, Radu le Bel, homme du sultan. Quant à la barbarie des représailles, elle semble avoir été largement partagée par les souverains de la région à l'époque, ce qui a permis à Victor Hugo le vers fameux où le sultan, ayant fait emmurer vifs vingt mille captifs valaques, fait "écrire sur le mur sombre / "Mourad, tailleur de pierres, à Vlad, planteur de pieux" ".

\section{BIBLIOGRAPHIE}

Bernard, Yvelise, Kaplan, Michel, Vincent-Cassy, Mireille, Zimmermann, Michel, (1993), Dictionnaire des biographies, 2. Le Moyen Âge, Paris : Armand Colin, 309 p.

Budai-Deleanu, Ion, (1969), Ţiganiada (Édition critique - Florea Fugariu ; Étude introductive Romul Munteanu), Bucarest : Editura Tineretului, vol. I, 325 p. \& vol. II, 326 p.

Cazacu, Matei, (1996), L'histoire du prince Dracula en Europe centrale et orientale ( $\mathrm{XV}^{e}$ siècle).

Présentation, édition critique et commentaire, Genève : Droz.

Chalcondili, Laonic, (1958), Expuneri istorice, editia Vasile Grecu, București : Ed. Academiei, 355 p.

Courthiade, Marcel (2004), « La Țiganiada de Ion Budai-Deleanu », dans Rajko Djuric et Marcel Courthiade, Les Roms dans les Belles-Lettres européennes, Paris : L'Harmattan, 190 p.

Ducas, Mihail, (1958), Istoria turco-bizantina (1341-1462), editia Vasile Grecu, Bucureşti : Editura Academiei, $500 \mathrm{p}$.

Mourre, Michel, (1986), Dictionnaire encyclopédique d'Histoire, Nouvelle édition, $k-m$, Paris : Bordas, $3200 \mathrm{p}$.

Panatescu, Panagioti, (1994), Interprétations roumaines, Bucarest : Editura enciclopedică, 257 p.

Stoiescu, Nicola, (1969), Vlad Ţepeş, Bucarest : Editura militara, 197 p. 


\section{NOTES}

1. De son nom roumain Ţara românească, mais aussi appelée en latin Uallachia - sous influence allemande, c'est-à-dire " pays étranger ».

2. Vlad III de Muntenie ou Vlad Ţepeş («l'Empaleur») ( 1430/1431, Schässburg/Sighişoara $\uparrow 1476$, Bucarest).

3. Le Diable, surnom en fait élogieux hérité de son père, lequel avait été nommé Dracul (le Dragon) par référence à l'Ordre du Dragon, offert en 1431 par l'empereur Sigismond de Luxembourg (*Nuremberg, 1368 - †Znaim, 1437), roi de Hongrie de 1387 à 1437), empereur germanique de 1411 à 1437.

4. Vlad II de Munténie ou Vlad Dracul (le Diable en roumain) ou Draculea (*vers 1390 - †1447), prince de Munténie de 1436 à 1442 et de 1443 à 1447.

5. C'est cette réputation que Victor Hugo lui connaissait: "Vlad, boyard de Tarvis, appelé Belzebuth, / Refuse de payer au sultan le tribut, / Prend l'ambassade turque et la fait périr toute / Sur trente pals, plantés aux deux bords d'une route ».

6. Ion Budai-Deleanu (* 1760, Cigmău, près de Hunedoara en Transylvanie - † 1820, Lviv, en Ukraine) achève en 1800 son œuvre complexe et de valeur universelle, comme l'a souligné M. Courthiade (Djurić, Rajko \& Courthiade, Marcel "Les Rroms dans les Belles-Lettres européennes", L'Harmattan, Paris, 2004, pp. 125-155), et paru en 1857-1877 sous le titre de La Ţiganiada. Il a publié La Ţiganiada sous le pseudonyme de Leonáchi Dianèu. Ion Budai-Deleanu connaissait bien les Rroms ainsi que leur origine : l'Inde, puisqu'il l'affirme dans l'introduction de son œuvre (fin du paragraphe 5 de la lettre de dédicace : « Noi sîntem din India, şi limba noastră să grăieşte acolo pănă în zioa de astezi; însă pentru aceasta Ţi-oi scrie de altă dată » ("Nous sommes venus de l'Inde, là-bas on parle notre langue (depuis notre départ) jusqu'à aujourd'hui ; mais ça je te l'écrirai une autre fois »). L'auteur avait connaissance de cette (re)découverte tout à fait récente, qui ne date que de la fin du XVIII ${ }^{e}$ siècle. Une telle intimité avec les Rroms, révélée dans ce poème héroïco-comique, ne pouvait réellement s'acquérir que par une fréquentation assidue de ce peuple, dont plusieurs auteurs estiment par ailleurs qu'il était issu.

7. Matthias I ${ }^{\text {er }}$ Corvin (* Koloszvar, Cluj, 1440 - † Vienne, 1490), roi de Hongrie (1458-1490). Fils de Jean de Hunyade, il fut emmené avec son frère aîné, en 1457, comme otage à Prague, sur l'ordre de Ladislas V le Posthume. Après la mort de Ladislas, il fut élu roi par les magnats hongrois (24 janv. 1458). Il eut aussitôt à maintenir l'indépendance hongroise à la fois contre l'Autriche et contre les Turcs. Un parti d'opposants ayant offert la couronne à l'empereur Frédéric III, il réussit à se rallier la noblesse, battit les troupes impériales et se fit restituer par l'empereur la couronne de Saint-Étienne (1462). Se retournant alors contre les Turcs, il soumit la Bosnie (1462) et leur enleva la Moldavie et la Munténie (1467). L'année suivante, il engagea la guerre contre son beau-père, le roi de Bohême Georges Podiebrad, et prit lui-même le titre de roi de Bohême (1469). Ce conflit se poursuivit jusqu'en 1469, et Ladislas II consentit à céder à Matthias Corvin la Silésie, la Lusace et la Moravie. Peu après, Matthias reprit les armes contre l'empereur Frédéric III, s'empara de la Styrie, de la Basse-Autriche et même de la ville de Vienne (1485). (Mourre, Michel "Dictionnaire encyclopédique d'Histoire, Nouvelle édition, $k-m$ ", p. 3000).

8. Cazacu, Matei, L'histoire du prince Dracula en Europe centrale et orientale (XVe siècle). Présentation, édition critique et commentaire, Droz, 1996, p. 30.

9. N. Stoicescu, Vlad Ţepeş, Ed. Militară, Bucureşti, 1969, p. 5.

10. Ibidem, p. 163.

11. Jiskra devait aider Vlad III à récupérer le trône de la Munténie. Mais le 26 novembre 1462 , près de Rucar, à Piatra Craiului (Königstein), Jiskra reçut l'ordre du roi Matthias Corvin de l'arrêter (Ibid., p. 150).

12. Ibid., p. 163.

13. Ibid., p. 181.

Cahiers balkaniques, 36-37 | 2008 
14. Mehemmed II (ou Mehmed II, Mahomet II, Mahomed II) (*1429/1430/1432 - †1481), sultan ottoman (1444/46-1451/81) surnommé al-Fatih (le Conquérant) vainqueur de Constantinople (29 mai 1453). Son père Murad II $\left({ }^{*} 1403 / 1404\right.$ - †1451) fils de Mehemmed I ${ }^{\text {er }}$ et petit-fils de Bayezid I ${ }^{\text {er }}$, sultan ottoman (1421/44-1446/51), abdiqua en 1444, en sa faveur, mais reprend le pouvoir en 1446. (Bernard, Yvelise; Kaplan, Michel; Vincent-Cassy, Mireille; Zimmermann Michel, Dictionnaire des biographies, 2. Le Moyen Âge, Armand Colin, Paris, 1993, p. 228.)

15. Chalcocondili L., Expuneri istorice, editia Vasile Grecu, Ed. Academiei, Bucureşti, 1958, 355 p.

16. Doukas, M. Istoria turco-bizantina (1341-1462), editia Vasile Grecu, Editura Academiei, Bucureşti, $1958,500 \mathrm{p}$.

17. L. Chalcocondili, op. cit. p. 283 et 289 , parle de 20.000 empalés, mais des Roumains, non pas des Turcs.

18. «En 1456 quand Vlad l'Empaleur commence son règne, la Munténie était obligée de s'acquitter d'un tribut appelé haraç correspondant à 10.000 pièces d'or. À cette époque cette somme d'argent était l'équivalent du prix de 200 villages, en sachant que le prix d'un village était de 45-50 pièces d'or. Pendant deux années, Vlad a apporté le tribut personnellement à la cour du sultan. Mais, à un moment donné, il a refusé de payer le tribut et il a rompu les traités avec les Turcs. Ce moment est identifié à l'année 1459, parce qu'en 1462 le sultan demandait le tribut pour les trois années passées. » N. Stoicescu, op. cit., p. 89.

19. L'auteur s'inspire de Chalcocondili, op. cit. pp. 283-284.

20. «Dans l'année 6970 le sultan demande au prince de Munténie d'apporter personnellement le haraç de 10.000 pièces d'or et 500 jeunes garçons. Le prince Vlad lui répond qu'il peut lui donner tout de suite les pièces d'or, mais qu'il ne peut pas lui donner les garçons et qu'il lui est impossible d'aller se prosterner devant le sultan. » Ducas, op. cit. p. 430.

21. Chalcocondili montre au contraire que Vlad n'était pas au courant de ce plan, et qu'il tombe dans le piège, mais qu'il se sauve grâce à son héroïsme.

22. Il se nommait en réalité Thomas Catabolites.

23. "Cette armée a été évaluée différemment par les sources de l'époque: Petru de Thomasiis évalue ses effectifs à 60.000 soldats, dont 25.000 janissaires, le chroniqueur byzantin L. Chalcocondili parle dans sa chronique d'une armée de 250.000 soldats et Tursun-bey de 300.000 soldats. Connaissant la tendance des chroniqueurs de gonfler les chiffres pour donner plus de grandeur aux événements de l'époque et si les effectifs de l'armée qui a conquis Constantinople ont été d'approximativement 80.000 soldats, il est possible que les effectifs minimums de l'armée turque aient été de 60.000 soldats, plus les troupes auxiliaires 20.000 soldats. Le sultan envoie aussi une armée par la mer, transportée dans 25 trirèmes et 150 autres navires, avec la mission principale d'occuper le port Chilia. L'armée de Vlad l'Empaleur était formée de tous les habitants capables de porter des armes à partir de 12 ans. Cette armée a été évaluée par Petru de Thomasiis à 22.000 soldats. Vlad l'Empaleur a séparé son armée en deux: une partie est restée pour combattre contre les Turcs (approximativement 15.000 soldats), et le reste (approximativement 7.000) a été envoyé à la frontière moldave. " N. Stoicescu, op. cit., p. 107.

24. L'exemple utilisé par Budai - Deleanu par l'entremise d'Argineanu est raconté par Chalcocondil : « [...] des soldats de l'empereur, cette nuit-là, ont arrêté un soldat de Vlad, ils l'ont conduit chez Mahmut; il lui a demandé qui il était et d'où il venait. Après quoi, il lui a demandé s'il savait où se cachait Vlad, le prince de la Dace. Il lui a répondu qu'il le savait, mais qu'il ne pouvait rien dire parce qu'il craignait le prince. Lorsqu'ils l'ont menacé de le tuer s'il ne disait pas tout ce qu'ils voulaient savoir, il a répondu qu'il était prêt à mourir à tout moment, mais il n'avait pas le courage de dire quelque chose concernant le prince Vlad. (L. Chalcocondil, op. cit. p. 289)

25. «De tous les chroniqueurs L. Chalcocondili décrit avec le plus de détails l'attaque de nuit de Vlad l'Empaleur. Selon Chalcocondil, Vlad aurait attaqué pendant la nuit avec 7.000 ou 10.000 cavaliers, provoquant une grande peur parmi les Turcs. L'attaque a était menée à la lumière de torches et de sonneries de cors. Même s'ils avaient très peur, les soldats turcs n'ont pas quitté leurs tentes parce que les hérauts du sultan leur intimaient l'ordre de rester sur place sous peine d'être tués par celui-ci. Le premier corps d'armée rencontrée a été l'armée asiatique qui a été vite obligée de s'enfuir. L'armée du prince Vlad, 
bien organisée et très disciplinée, avançait avec le but d'atteindre la tente du sultan et de le tuer. Mais par erreur ils sont arrivés devant les tentes des vizirs Mahmud et Isaac où l'armée roumaine a livré une grande bataille sans subir de pertes importantes. Cette méprise a permis aux gardes du sultan de se préparer pour faire face aux attaques des Roumains. Au lever du jour, les Roumains commencent la retraite. Si le sultan avait été tué pendant cette attaque de nuit, l'armée turque aurait était entièrement démoralisée et peutêtre les pertes auraient-elles été plus grandes. » N. Stoicescu, op. cit., pp. 118, 119.

26. Cette strophe est accompagnée de la note : «Ceci est à comprendre de manière allégorique, c'est-à-dire que Dieu a permis à Vlad de comprendre qu'il était vain de continuer à perdre des hommes contre les Turcs quand, de la résistance du pays et de la puissance des Turcs, il apparaît que Dieu n'avait pas encore décidé de soustraire le pays à la servitude » Budai-Deleanu, I., Ţiganiada (Édition critique - Florea Fugariu; Étude introductive - Romul Munteanu), Editura Tineretului, București, 1969, vol. II, p. 265.

27. Cette étude comporte dans les notes la traduction de nombreux passages du livre de BudaiDeleanu; ce sont ces traductions qui sont citées ici.

\section{RÉSUMÉS}

La Ţiganiada d'Ion Budai-Deleanu, un des pères de la langue roumaine moderne, est une épopée placée dans la Munténie du XV $\mathrm{XV}^{\mathrm{e}}$ siècle et structurée sur deux plans : d'une part, un plan comique créé par le pittoresque du campement des «Tsiganes », mobilisés pour combattre les Turcs par le prince Vlad l'Empaleur, surnommé Dracula, et, d'autre part, un plan héroïque constitué par les actions de ce prince et son armée. La promesse d'émancipation des Rroms esclaves, avec attribution d'un territoire, en échange de leur alliance au combat est une pure fiction et même un anachronisme pour le $\mathrm{XV}^{\mathrm{e}}$ siècle. Mais à travers cette idée, Budai-Deleanu se révèle visionnaire et partisan du mouvement d'abolition de l'esclavage des Rroms qui n'arrivera à ses fins que le 20 février 1856.

En raison de son insoumission entêtée au sultan Mahomet II, son courage et ses capacités stratégiques, Vlad l'Empaleur devait rester dans l'histoire roumaine comme un patriote qui voulait protéger son pays contre l'expansion ottomane - même s'il est plus connu à l'étranger sous la caricature d'un vampire.

La Ţiganiada doit être appréciée avant tout comme une allégorie où le plan de la fiction remplace souvent le plan réel. Mais l'auteur, chargé d'un idéal patriotique, nous présente aussi le plan réel avec ses particularités historiques et nationales. La lutte opiniâtre de la petite armée roumaine formée de paysans et de boyards permet de retarder l'avancée des Turcs vers l'Europe centrale. Elle laisse ainsi le temps nécessaire à l'Europe de se préparer et de livrer des batailles dans des périodes plus favorables, avec des armes plus puissantes et plus efficaces que celles des Turcs.

L'image des Turcs y est en tous points conforme à leur réputation, largement répandue dans les Balkans. Cruauté, perfidie, corruption et absence de compassion les caractérisent d'un bout à l'autre de l'œuvre, l'auteur montre cependant que ces maux n'auraient pu s'exercer sans les bassesses des boyards - voire le propre frère de Vlad, Radu le Bel, homme du sultan.

Triganiada of Ion Budai-Deleanu, one of the fathers of the modern Romanian language, is an epic placed in the 15th century Muntenia and structured on two plans: one a comical plan created by the picturesque quality of the "Gypsy" camp, mobilized by the prince Vlad the Impaler, named Dracula, to fight the Turks, and the other a heroic plan formed by the actions of this prince and 
his army. The promise of the emancipation of the Roms slaves, with the assignment of territory, in exchange for their alliance in combat is a pure fiction and even an anachronism for the 15th century. But with this idea Budai-Deleanu shows himself to be a visionary supporter of the movement for the abolition of slavery of the Roms which was not concluded until February 20th 1856. Because of his obstinate rebelliousness against Sultan Mahomet II, his courage and his strategic capacity, Vlad the Impaler must stand in Romanian history as a patriot who wanted to defend his country against Ottoman expansion, even if he is better known elsewhere in the caricature of a vampire.

The Tiganiada must be appreciated above all as an allegory in which fictional level often replaces the real one. But the author, driven by a patriotic ideal, also presents the representation of reality with its historical and national peculiarities. The stubborn struggle of the small Romanian army made up of peasants and nobles allowed the Turkish advance towards central Europe to be retarded. It also gave Europe the time required to prepare and to conduct battles at the most favorable time, with armies more powerful and effective than the Turks'. The image of the Turks conforms in all respects to their reputation, largely gained in the Balkans. Cruelty, perfidy, corruption and lack of compassion characterize them from start to finish of the work, the author shows however that these evils could not be exercised without the degradation of the nobles indeed the true brother of Vlad, Radu le Bel, the sultan's man.

\section{INDEX}

Mots-clés : littérature roumaine, Haraç/kharadj, Budai-Deleanu Ion (1760-1820), Budai-Deleanu Ion (1760-1820) , Munténie, Munténie, Vlad III l'empaleur (1431-1476), Tiganiada, épopée, Rroms, Ottomans dans la littérature

Keywords : rumanian litterature, Tiganiada, Vlad, Budai-Deleanu Ion (1760-1820), fifteenth century, Ottoman empire

Thèmes : Littérature

Index chronologique : Empire ottoman, quinzième siècle

motsclestr Romanya, Osmanlı İmparatorluğu

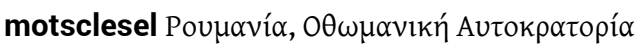

Index géographique : Roumanie

motsclesmk ОТОМАНСКАТА ИМПЕРИЈА, РОМАНИЈА 